\title{
Human-Internet Interaction: A Potential Platform towards Promotion of Integrated Pest Management Technology in India
}

\author{
Shubhaom Panda* and Jagadish Aditya Dinakar \\ Institute of Agri Business Management, Swami Keshwanand Rajasthan Agricultural \\ University, Bikaner, 334006, India \\ *Corresponding author
}

\begin{abstract}
A B S T R A C T
In the world, the number of internet users has blown up from only 413 million in 2000 to 3.4 billion in 2016. China and India grab the top two shares despite of having only 50 and 26 percent online internet users, respectively. Internet usage in India has been increased from 242.92 million during 2015 to 390.9 million during 2018. Out of all search engines, Google comprises of more than 91 per cent of users, worldwide. Till August 26, 2020, the interest of people in Punjab regarding IPM technology, is highest, followed by Odisha, Gujarat, Rajasthan and Tamil Nadu. In the year, 2019, the searches in the Google on the IPM topic have been highest in Punjab, followed by Haryana, Madhya Pradesh, Uttar Pradesh and Maharashtra. According to "Socialmention" website, the sentiment of people using online platform to show their emotion regarding IPM is mostly neutral, followed by positive and negative sentiments. "Integrated Pest Management" as a term has been searched 3600 times, mostly by people of age group 55-64 during a month in real-time, according to "ubersuggest". It was found that, during 2018, 21 percent of the Indian internet users used their smart phones to visit a social network, while 16 percent of the users watched videos. Facebook, the largest social media platform in the world, had 2.4 billion users during 2019. Regarding IPM technology, the websites like Google trends, Socialmention, Ubersuggest, etc. can be used for nowcasting and forecasting about the current thoughts, sentiments, practice, likings, etc. of the public in real-time basis. Facebook as the leading social network, should be utilized to promote IPM technology in a better way.
\end{abstract}

\section{Keywords}

IPM, Internet, Online Searching Trend, Facebook

\section{Article Info}

Accepted:

17 August 2020

Available Online:

10 September 2020

\section{Introduction}

The Internet and social media are becoming more significant in providing information resources to marketers and advertisers regarding what consumers are thinking and saying about their brands, products and campaigns. The Internet was primarily a oneway medium proposed for reading, in which the most users could only browse the available websites. There were a very few content creators as compared to the majority of internet users (Cormode, 2008). In early 2000, the progress of technology and the development of applications allowed two-way communication with the users, which led to the transformation of the internet user's role, turning from a passive viewer to an active 
participant. This period is known as Web 2.0. Compilation and analysis of posts on social media platform open up endless possibilities for many professionals. In the era of internet, Integrated Pest Management as a mean to Sustainable Agriculture finds its way to be promoted through this article. The model of sustainable agriculture mostly emphasizes on enhancing the efficacy of agrochemical usage by the implementation of integrated pest management (IPM) technology. It substitutes various inputs and practices with effective alternative technologies involving low energy utilization and plant/animal integration (Alam et al., 2016; Lechenet et al., 2014; Seufert et al., 2012). The demand for integrated pest management technology in the world was valued at USD 91.8 billion in 2016, which was expected to grow at a compound annual growth rate (CAGR) of 5.8 per cent, valued at USD 151 billion by 2025. With an anticipated CAGR of 6.4 per cent from 2017 to 2025, the Asia pacific region was expected to witness the fastest growth in IPM technology. In India, the plant protection division performs regulatory, monitoring, surveillance and human resource development functions through a scheme called Sub-Mission on Plant Protection and Plant Quarantine (SMPPQ) under Green Revolution (Krishonnati Yojana). In this regard, the Destructive Insects \& Pests Act, 1914 and the Insecticides Act, 1968 provide the legal framework for the regulatory function.

The Directorate of Plant Protection Quarantine \& Storage (DPPQ\&S), Faridabad, Haryana was established in the year 1946 on the suggestion of Woodhead Commission as a top organization for advising the Indian Government and various state governments on every matters related to plant protection. National Institute of Plant Health Management (NIPHM) was established at Hyderabad in 1966 as a component under SMPPQ and it provided pest management capacity building programme to state officials and farmers. Government of India has adopted IPM as the fundamental principle and main plank of plant protection in the overall crop production programme since 1985. IPM activities are funded under central sector plan scheme 'Strengthening and Modernization of Pest Management Approach (SMPMA)' under National Mission on Agricultural Extension and Technology (NMAET) of the DPPQ\&S (DAC\&FW, 2020).

The Indian Council of Agricultural Research (ICAR) established the National Centre for Integrated Pest Management (NCIPM) on 12th February, 1988 during VIIth Five Year Plan at Faridabad, Haryana, which was later shifted to Lal Bahadur Shastri Centre for Advanced Research in Plant Protection and Biotechnology, IARI Campus, New Delhi. NCIPM targets for covering at least 30 per cent of the arable area under IPM by 2030, through a nation-wide network of the stakeholders on major crops including cotton, rice, pulses, oilseeds, sugarcane, horticultural crops, and organic / protected export oriented cultivation (NCIPM, 2011). Central Integrated Pest Management Centres (CIPMCs) had been established in 1992 by merging all Central Plant Protection Stations (CPPS), Central Surveillance Stations (CSS) and Central Biological Control Stations (CBCS). Since 2006, seed treatment campaign is organized every year by the department during Kharif and Rabi seasons that include state departments of agriculture as well as the CIPMCs. Also large scale adoption of Grow Safe Food (GSF) campaign had also been taken up to curb the misuse of chemical pesticides in agriculture. As on date, 35 CIPMCs are working in the country. Pesticide residue monitoring at the national level scheme has been working successfully since 2005, as a central sector scheme to gather, collate and analyze sample data and information on the existence of certain 
pesticide residues in agricultural commodities at market yards and farm gates, in a centralized manner. Samples are drawn from agricultural and food products including animal produce which are then sent for testing to 25 participating laboratories accredited by 'National Accreditation Board of Central Government and Agricultural Universities'. As a remedial action for appropriate and judicious use of pesticides with an integrated pest management approach, the gathered data regarding pesticide residue is then shared with various state governments.

Apart from organizing farmers field schools (FFSs), the CIPMCs carry out disease/pest monitoring, production, release and conservation of bio-control agents. Till 2020, NCIPM with collaboration of NIPHM, has developed 87 numbers of IPM packages of practices of major crops for disease/pest management. Because of intervention of CIPMCs, crop yield increased from 6.72 to 40.14 per cent in rice and 22.7 to 26.63 per cent in cotton in IPM fields compared to nonIPM fields, chemical pesticide sprays were reduced to the extent of 50 to 100 per cent in rice and 29.96 to 50.5 per cent in cotton, use of bio-pesticides and neem based pesticides increased from 123 metric tonnes during 1994-95 to 7,682 metric tonnes (technical grade) during 2018-19 and over all consumption of chemical pesticide in the country reduced from 61,357 metric tonnes (technical grade) during 1994-95 to 49,438 metric tonnes (technical grade) during 201819 (DAC\&FW, 2020). In 2016, a collaborative project of Space Application Centre (SAC, ISRO), Ahmedabad and ICARNCIPM on 'Forewarning of major crop pests on spatial scale for their integrated management' has been launched which is basically a satellite-based value-added agromet products for early warning to farmers. PESTPREDICT, an android mobile application for pest forewarning, has been developed by ICAR-NCIPM using Java and Android through information and communication technology (ICT) based pest surveillance under National Innovations in Climate Resilient Agriculture (NICRA) in 2016. Also in the same year, 'Insecticide Calculator' for Smart phones for the management of sap feeders of cotton has been launched. Various mobile Insecticide and Fungicide Calculators (IFCs) have been developed using Core Java, Eclipse platform and Android software to provide information on chemical and biological insecticides and fungicides together in respect of twelve crops including cotton and chickpea. NCIPM is implementing 'Mera Gaon Mera Gaurav' (MGMG) a flagship programme involving five multidisciplinary team of scientists who provide awareness on IPM in 27 adopted villages, to address and solve crop raising and pest management (NCIPM, 2019). For the implementation of IPM technology, under Rashtriya Krishi Vikash Yojana (RKVY), the government is also providing funds to the states. Maharashtra leads in receiving funds from the government for IPM followed by Gujarat, Madhya Pradesh and others, as it can be well observed from the table 1 .

Internet has become an essential element of life. With the help of internet, the world is running very fast. Clement (2020) discussed that during 2019, the number of internet users worldwide was 4.13 billion, which was more than 3.92 billion in 2018. Easier accessibility of computers, the rejuvenation of nations around the world and an increased use of smart phones has given people the scope to use the internet frequently and with convenience. By 2017, there were about 772 million internet users in China and 312 million internet users in the United States. Because of having infrastructural issues, broadband internet usage has not been successful in many countries and, developing online markets relied robustly on mobile 
connections. Consequently, global mobile data traffic is set to exceed 77 exabytes per month in 2022, up from 11.5 exabytes per month by 2017 . Social networking is the most popular online activities and Facebook is the most liked online social network. There were more than 2.2 billion monthly active Facebook users, resulting for almost half of worldwide internet users, as of the third quarter of 2018. The most popular reasons for internet usage has been, connecting with close beings, stating opinions, entertainment and online shopping, etc. Keelery (2020) revealed that in India, out of 483 million internet users, 390 million users accessed the internet through their mobile phones, during 2018. Estimates suggest that this figure would reach over 500 million by 2023. Use of mobile phones had resulted more than 73 per cent of India's total web-traffic, in 2019. The increased availability of cheap smart phones from 2010 and more investment in telecom sectors in India with new telecom spectrum licenses, were the two major reasons for the growth of mobile internet users in India. In September, 2016, Reliance Jio entered the Indian market, providing customers with cheap 4G, high speed data plans with free voice calling. Within just six months of its launch, the company had attracted more than 100 million customers and by the end of 2019, it had become the highest wireless telecom subscriber in India, leading to a cumulative growth in mobile internet accessibility as well as affordability. Keelery (2020) projected that by 2023, the number of Facebook users in India is expected to reach 444.2 million, up from 281 million in 2018 indicating a steady growth in the social media platform's user base. Social networking, one of the main online activities, which shows healthy projections for the coming years, as the number of social network users worldwide is forecasted to grow from 2.14 billion in 2015 to nearly three billion in 2020 .

\section{Materials and Methods}

This study is based on descriptive research design. For the study, secondary data has been used. The data have been collected from various journals, websites, and other likely sources. The data have been analyzed by using MS office 2007. Statistical tools like percentages and Compound Annual Growth Rate (CAGR) have been used wherever found suitable to discuss.

\section{Results and Discussion}

Under this head, the status of internet in India and the statistics regarding internet usage regarding IPM have been reviewed.

\section{Status of Users of Internet in India}

Internet leads to success in every parts of life. The importance of internet in present context has been discussed in the figure 1 as below. The percentage of use of internet worldwide is shown as below, where India has 20-30 per cent of total population who use internet.

The figure 1 shows the share of the population accessing the internet in the world, country wise. This figure 1 shows the percentage resulting from the usage of internet in the last three months at the time of study. The internet use might have been possible through any medium like computer, mobile phones, etc. The figure shows the less percentage in the developing countries but they are increasing with an increasing rate over the world. The internet has become one of the most transformative and growing technologies. Worldwide, the number of users of internet had increased from only 413 million during 2000 to about 3.4 billion by 2016. The one billion barriers had been crossed in 2005. For the last five years, an average of 640,000 people was going online for the first time, every day. 
From the figure 2, it can be well observed that, Asia leads in internet usage over the world, followed by other regions. China and India grab the top two shares despite of having just 50 and 26 percent online internet users, respectively. The top six countries by users (and the only countries with over 100 million) in 2016-17 were China (765 million), followed by India (391 million), United States (245 million), Brazil (126 million), Japan (116 million), and Russia (109 million). The internet has been growing at an increasing rate in many developing countries like India, Bangladesh, Ghana, Malawi, etc. which have doubled the number of internet users in the last 3 years.

The figure 3 shows the increasing trend of internet use in India. In this figure, internet users are the individuals who had used the internet from any location, in the three months before the research. The internet could be used via any medium viz. a computer, mobile phone, etc. The rise of mobile phones has become a truly disruptive technological advancement in India.

It can be well observed from the figure 4 that, the internet usage has been increased from 242.92 millions in 2015 to 390.9 million in 2018. It also estimates an increase to 500.9 millions of mobile internet users by 2023 . Using smart phones have increased the internet usage but the popular activities used for using internet are as shown in the figure 5.

\section{Human-Internet Interaction about IPM in India}

Human beings use internet a lot now a days. With the changing world, the use of search engines has been inevitable in the day to day life. Out of all search engines, Google comprises of more than 91 per cent of users, worldwide. This search engine feeds the population of the world and enriches them with knowledge and information. As it can been seen from the figure 5 below, Google leads as the best choice for the internet uses of the world, followed by Bing, Yahoo, Baidu, Yandex, and others

Government of India tries to promote IPM technology in a lot of ways. NCIPM has many mobile based applications on different IPM modules for different crops. But, still farmers are not installing and following the technology. The reasons may be the medium chosen by the government to promote such type of mobile based applications. The government agencies should promote the IPM technology by taking prior information about the people interested. The regions have to be selected where the interest regarding IPM is high and creating those areas as the reference regions for other to accept. The use of Google trends and facebook can be a major breakthrough in this regard. If the present scenario of interest of IPM technology will be checked, then Google trends will be a great choice to see the human-internet interaction at different locations of the country. The figures below show the states where people are more interested in IPM related topics than other states.

From the figure 6, it is clear that, till August 26, 2020, the interest of people in Punjab regarding IPM technology, is highest, followed by Odisha, Gujarat, Rajasthan and Tamil Nadu. A score of 100 always represents the highest relative search volume. The resulting numbers are then scaled on a range of 0 to 100 based on a topic's proportion to all searches on all topics.

In the year, 2019, the searches in the Google on the IPM topic have been highest in Punjab, followed by Haryana, Madhya Pradesh, Uttar Pradesh and Maharashtra. In the present context, India stands second in internet usage. There are websites, which directly gives the 
sentiment analysis for a particular term on the real time. Another example of such type of website is socialmention.com, which applies natural language processing (NLP) to explore online mentions and finds out the feelings behind the message. Social Mention is a simple platform to use social media search tool which aggregates the user generated content across the internet, into a single stream of information. Social media sentiment analysis identifies whether an user reacts positively, negatively, or is neutral to messages in any online platform (Fig.7). The analysis gives four basic outputs viz. strength, sentiment, passion and reach. Strength describes how likely it is that a particular thing is being discussed in social media. Sentiment allows identifying general attitudes towards anything, whether positive, negative, or neutral. Passion measures the frequency with which people post on social channels and talk about the item, searched. Reach measures the awareness and the range of influence about anything searched, by which the amount of users that could have seen the post, can be analyzed. Figure 8 shows the sentiment analysis of IPM in India.

From the figure 8 , it is clear that the sentiment of people using online platform to show their emotion regarding IPM is mostly neutral, followed by positive and negative sentiments. It can been observed that how the internet is important in building confidence among the public.

There is also another free website, named as ubersuggest of neilpatel.com, to find out the most used keywords for a particular topic during a month in real time. The table 2 given below provides us the real time search volumes in India for the keyword "Integrated Pest Management". "Integrated Pest Management" as a term has been searched 3600 times during a month in real time according to "ubersuggest".

The figure 9 outlines that, the maximum number of searches are made by people of age between 55-64, followed by 45-54 and 35-44.

Table.1 Approval of total funds for IPM technology under RKVY in major states (2012-13 to 2018-19)

\begin{tabular}{|l|c|}
\hline States & Total funds for IPM (in crores) \\
\hline Maharashtra & 259.46 \\
\hline Gujarat & 115.27 \\
\hline Madhya Pradesh & 48.32 \\
\hline Odisha & 35.54 \\
\hline Andhra Pradesh & 30.27 \\
\hline Punjab & 27.07 \\
\hline Jharkhand & 26.60 \\
\hline Haryana & 26.27 \\
\hline Telangana & 20.53 \\
\hline West Bengal & 16.06 \\
\hline Others & 71.71 \\
\hline India & 677.10 \\
\hline
\end{tabular}

Source: Annual Report 2018-19 \& 2017-18, Department of Agriculture, Cooperation \& Farmers Welfare, Ministry of Agriculture \& Farmers Welfare, Government of India 
Table.2 Top Ten Keywords searched relating to IPM in real-time

\begin{tabular}{|c|l|c|}
\hline S.N. & \multicolumn{1}{|c|}{ Keyword } & Search Volume* \\
\hline $\mathbf{1}$ & integrated pest management & 3600 \\
\hline $\mathbf{2}$ & integrated pest management def & 590 \\
\hline $\mathbf{3}$ & integrated pest management define & 590 \\
\hline $\mathbf{4}$ & integrated pest management pdf & 480 \\
\hline $\mathbf{5}$ & integrated pest management ppt & 320 \\
\hline $\mathbf{6}$ & integrated pest management methods & 320 \\
\hline $\mathbf{7}$ & integrated pest management tools & 260 \\
\hline $\mathbf{8}$ & what is integrated pest management & 260 \\
\hline $\mathbf{9}$ & integrated pest management slideshare & 260 \\
\hline $\mathbf{1 0}$ & integrated pest management definition & 210 \\
\hline
\end{tabular}

*The number of searches the particular keyword during a month

Source:https://app.neilpatel.com/en/ubersuggest/overview?keyword=integrated $\% 20 \mathrm{p}$ est $\% 20$ management\&locId=2356\&lang=en, retrieved as on 30.08.2020

Fig.1 Share of the population using the Internet, 2017

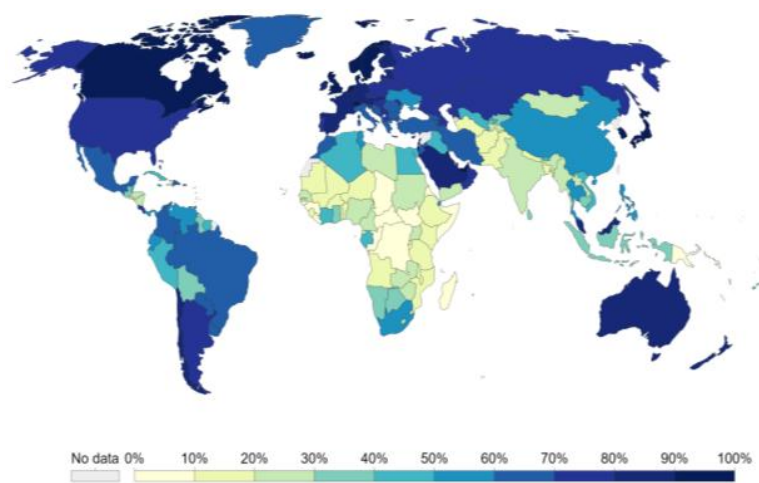

Source: https://ourworldindata.org/internet, retrieved as on 30.08.2020

Fig.2 World Internet Users (In Crores) till 2020*

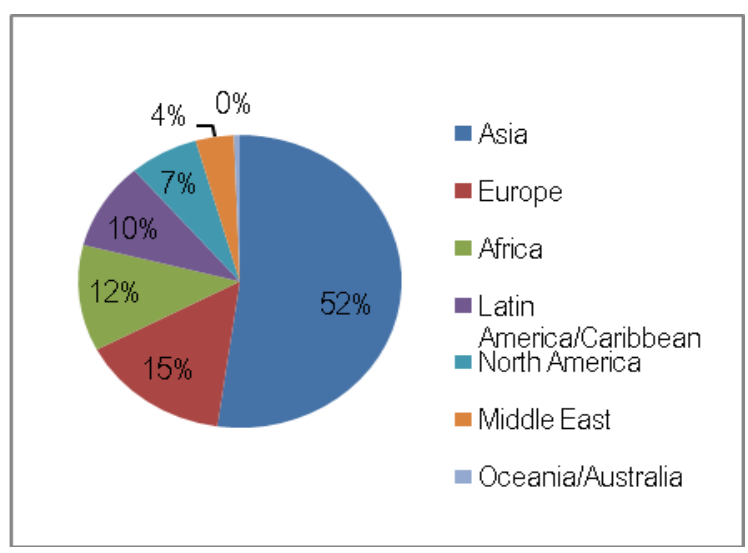

*Up to June 30, 2020

Source: https://www.internetworldstats.com/stats.htm, retrieved as on 30.08 .2020 
Fig.3 Number of internet users in India (1990-2016)

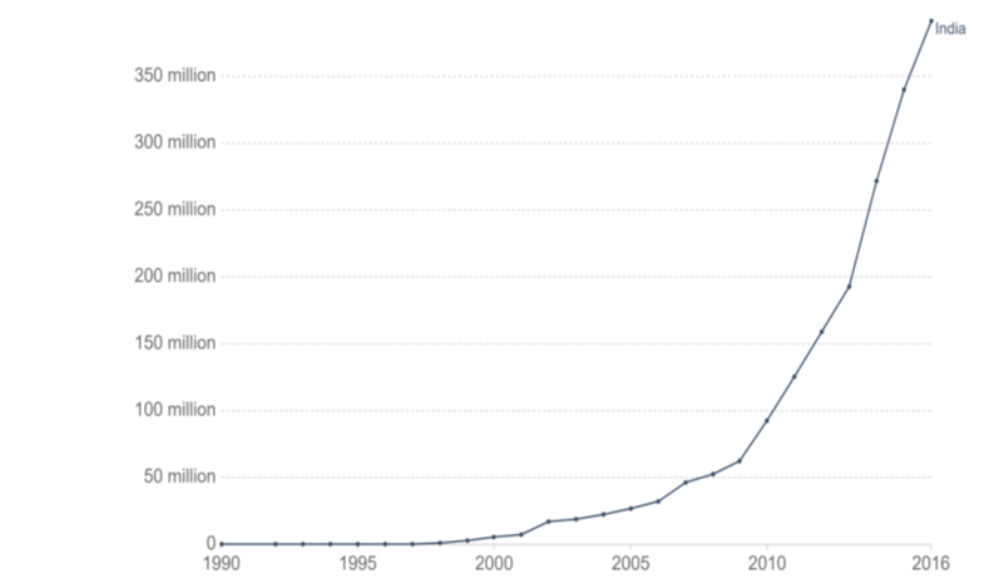

Source: https://ourworldindata.org/internet, retrieved as on 30.08.2020

Fig.4 Number of mobile phone internet users in India from 2015 to 2018 with a forecast until 2023

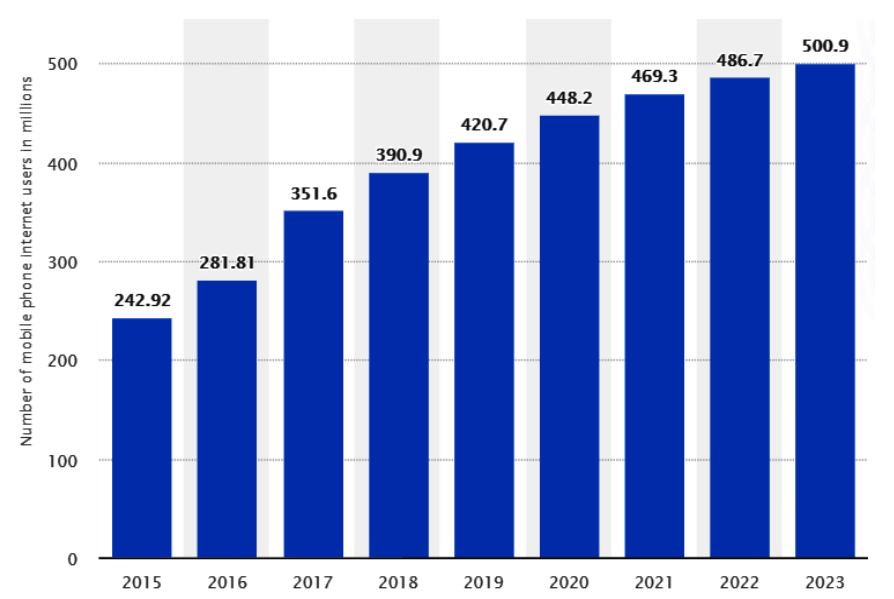

Source: Source: https://ourworldindata.org/internet, retrieved as on 30.08 .2020

Fig.5 Search engine market share in 2020

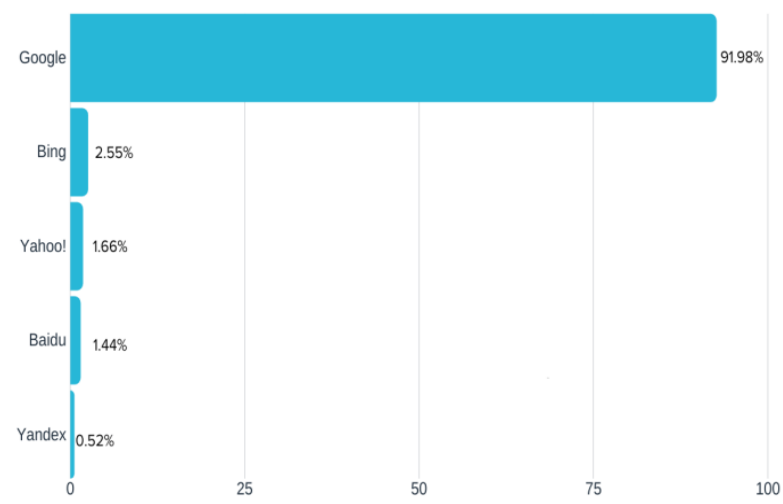

Source: https://www.oberlo.com/statistics/search-engine-market-share, retrieved as on 29.08 .2020 
Fig.6 Interest of people among states regarding IPM (2020)

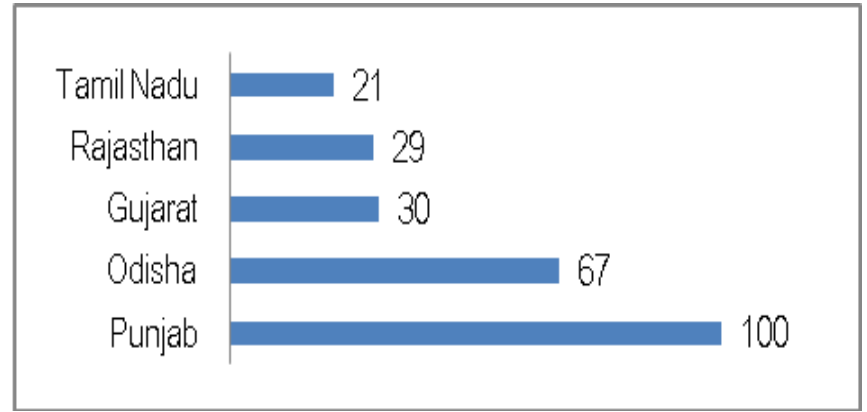

Source: Google Trends, retrieved as on 26-08-2020

Fig.7 Interest of people among states regarding IPM (2019)

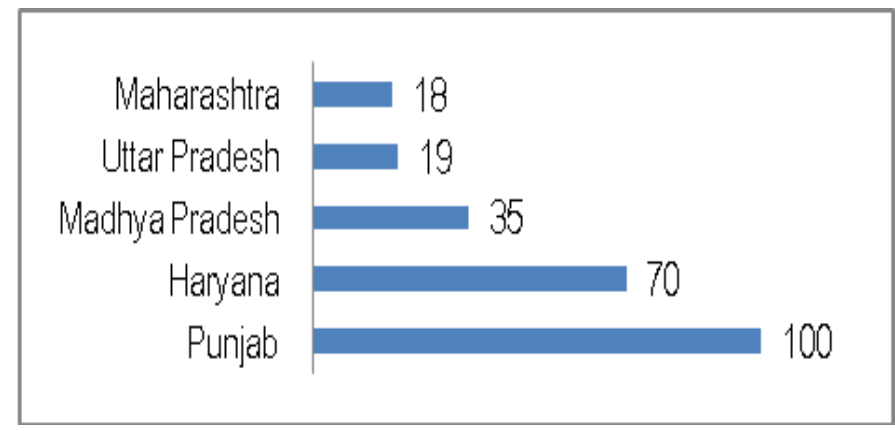

Source: Google Trends, retrieved as on 26-08-2020

Fig.8 Real-Time Sentiment Analysis of IPM

\section{socialnention*}

\section{$0 \%$ \\ strength \\ $13 \%$ \\ passion}

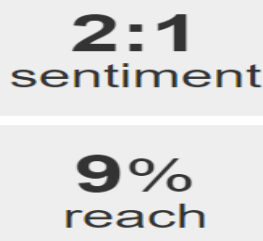

Sentiment

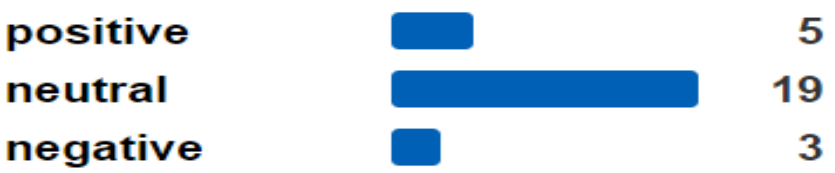

Source:http://socialmention.com/search?q=integrated+pest+management+\%28IPM\%29\&as_epq=integrate $\mathrm{d}+$ pest+management\&as_eq=\&t=all\&l=India\&tspan=\&num=100\&lang=\&sort_by=date\&as_fu=, retrieved as on 30.08.2020 
Fig.9 Searches done by different age group for IPM in Real time

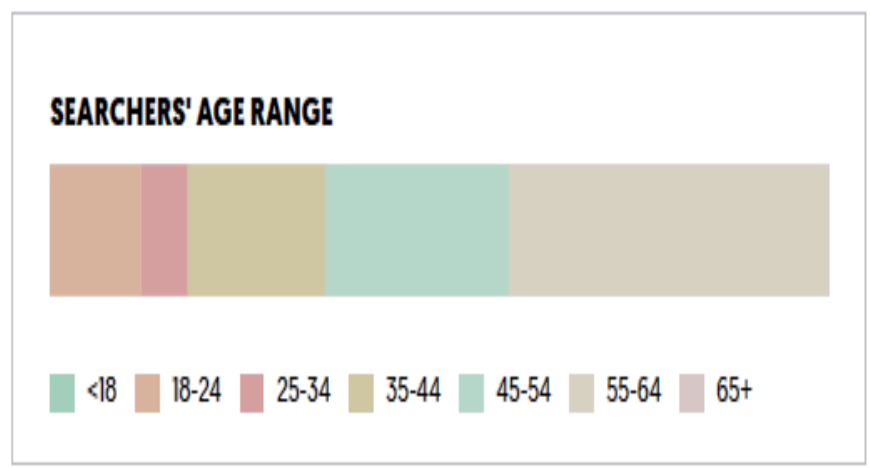

Source:

https://app.neilpatel.com/en/ubersuggest/overview?keyword=integrated $\% 20$ pest $\% 20$ management\&locId= 2356\&lang=en, retrieved as on 30.08.2020

Fig.10 Most popular Smartphone activities in India as of January, 2018

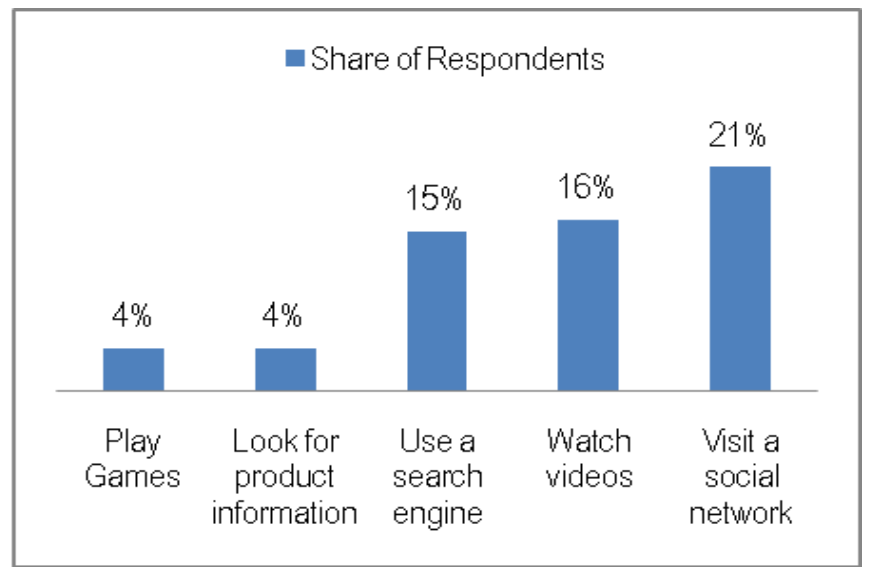

Source: https://ourworldindata.org/internet, retrieved as on 30.08.2020

Fig.11 Facebook users in India till February, 2020

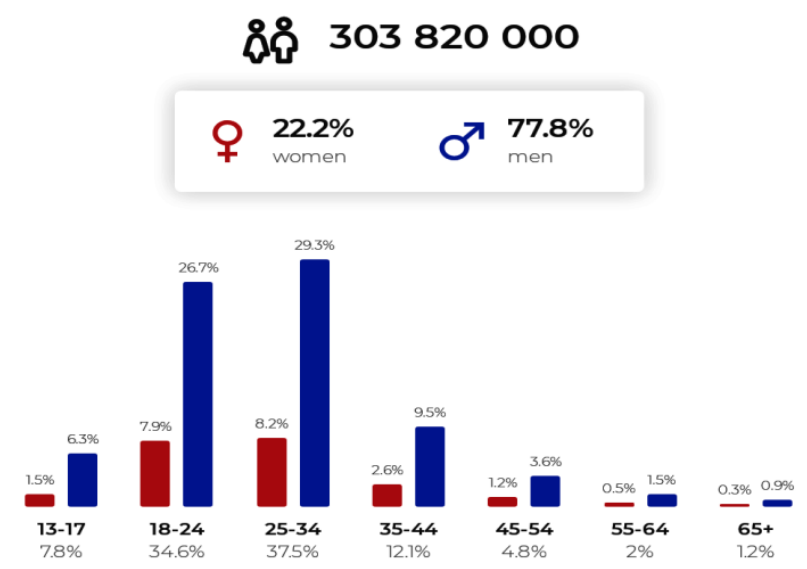

Source: https://napoleoncat.com/stats/facebook-users-in-india/2019/02, retrieved as on 30.08.2020 
In India, social networking websites have a great importance in this. The importance of social networks in India has been discussed as below. The use of social networks can be a strategy to promote IPM technology among the youth of the nation.

As, it can be seen from the figure 10, during 2018, 21 percent of the Indian respondents used their smart phones to visit a social network, while only 16 percent watched videos, followed by others. In 2019, Facebook, has become the largest social media platform in the world having more about 2.4 billion users. In the same year, other social media platforms like Youtube and Whatsapp also had more than one billion users, each. There are about 7.7 billion people in the world and out of which about 3.5 billion of users had been found online. This means social media platforms are being used by one-in-three people in the world, and more than two-thirds of all internet users. Facebook also owns three of the most popular social networks in the world, WhatApp, Facebook Messenger and Instagram. Social networking, one of the main online activities, shows healthy predictions for the near future. The Facebook accounts for the majority of these users, as the social network is the most popular social network the country. There were more than 195 million Facebook users in India by May 2016, against about 191 million in the U.S. and 90 million in Brazil. India is the country with the largest Facebook user base in the world. The number of Facebook users in India is forecasted to continue to grow in the near future, and is estimated to have more than 320 million by 2021. In general, young people are more likely to use social media than older people. But some platforms are much more popular among younger people. The figure 11, as shown below is the plot of the breakdown of social media used by different age groups and genders in India.
The figure 11 shows that, the maximum users within the age group of 18-34 are using facebook, which can be said that, the youth of the country is following social network largely.

\section{Suggested Strategies for promotion of IPM on online platform}

As, it is observed from the above results and discussion, it is clear that, youth of India spends lot of time in facebook. So, the government should look after the platform and may consider following suggestions for the promotion and dissemination of IPM technology in India.

- The government agencies working on IPM technology should exploit online platforms like Google trends, social mention.com, ubbersuggest, etc to get a knowhow about the current state of minds about IPM technology among the internet users in India.

- Government of India or NCIPM should create an authenticated or authorized facebook community for IPM technology.

- Language has always been a barrier in adoption of IPM technology. At least, National language, Hindi, should be considered for the information dissemination through facebook platform.

- Webinars on IPM should be organized at regular intervals on facebook platform.

- Importance should be given to the issues, challenges faced by the IPM adopters, through comments, likes and dislikes in the facebook page.

- All Government reports on IPM should be made available in the facebook community page.

- Success Stories of IPM adopters should be published in the facebook community page.

- The particular community should be 
promoted among the facebook users throughout the nation by using facebook ads.

In conclusion from the results above, it is very clear that there are many websites to check after the current trend of human-internet interaction regarding Integrated Pest Management in the country. The states which have interest on IPM technology should be promoted more about IM and may be taken as the role models for other states to opt. The high use of mobile internet shows that, there should be friendly and updated online applications linked with the current trends of the country, so that the interest of the public can be matched altogether. The internet should be utilized to a great extent for the betterment of the agriculture and the farming society at large. The websites like Google trends, Socialmention, Ubersuggest, etc. can be used for knowing the current thoughts, sentiments, practice, likings, etc. of the public in real-time basis. The nowcasting and forecasting can be well implemented with the knowhow of the interest of the public. The human-internet interaction has much more to see and the agencies should take the benefit out of that for the society at large.

Application of research: Future application of internet for promotion of Integrated Pest Management in India

Research Category: Review paper on Promotion of IPM technology

Author Contributions: All author equally contributed

Author statement: All authors read, reviewed, agree and approved the final manuscript

Conflict of Interest: There is no conflict of interest.
Ethical approval: This article does not contain any studies with human participants or animals performed by any of the authors.

\section{References}

Alam, M. Z., Crump, A. R., Haque, M. Manjurul., SirajulIslam, M., Hossain, E., Hasan, S. B., Hasan, S. B., \& Hossain, M. S. (2016). Effects of Integrated Pest Management on Pest Damage and Yield Components in a Rice Agro-Ecosystem in the Barisal Region of Bangladesh. Frontiers in Environmental Science, 4(22), 1-10.

Clement, J. (2020). Global number of internet users 2005-2019. https://ourworldindata.org/internet

Cormode, G. (2008). Key differences between Web 1.0 and Web 2.0. First Monday, 13

DAC\&FW (2020). Chapter 22, Sub-Mission on Plant Protection and Plant Quarantine (SMPPQ). Annual Report, 2019-20, Directorate of Agriculture, Cooperation \& Farmers' Welfare, Ministry of Agriculture, Government of India, 203-210.

Keelery, S. (2020). Mobile phone internet users in India 2015-2023. https://ourworldindata.org/internet

Keelery, S. (2020). Number of Facebook users India 2015-2023. https://ourworldindata.org/internet

Lechenet, M., Bretagnolle, V., Bockstaller, C., Boissinot, F., Petit, M. S., Petit, S., \& Munier-Jolain, N. M. (2014). Reconciling pesticide reduction with economic and environmental sustainability in arable farming. PLOS One, 9(6), 1-10.

NCIPM (2011). Consolidation of Plant Protection, Vision 2030, National Centre for Integrated Pest Management, ICAR, New Delhi, 9-11.

NCIPM (2019). Annual Report 2018-19, ICAR- National Research Centre for 
Integrated Pest Management, 1-128.

Seufert, V., \& Ramankutty, N, \& Foley, J. A. (2012). Comparing the yields of organic and conventional agriculture. Nature. 485, 229-232.

\section{How to cite this article:}

Shubhaom Panda and Jagadish Aditya Dinakar. 2020. Human-Internet Interaction: A Potential Platform towards Promotion of Integrated Pest Management Technology in India. Int.J.Curr.Microbiol.App.Sci. 9(09): 2132-2144. doi: https://doi.org/10.20546/ijcmas.2020.909.266 